\title{
The effect of chemotherapy on health-related quality of life in mesothelioma: results from the SWAMP trial
}

D T Arnold ${ }^{*, 1}$, C E Hooper ${ }^{1,2}$, A Morley ${ }^{2}$, P White ${ }^{3}$, I D Lyburn ${ }^{4}$, J Searle ${ }^{4}$, M Darby $^{5}$, T Hall $^{6}$, D Hall ${ }^{4}$,

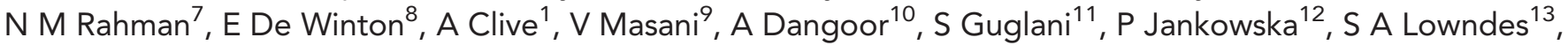
J E Harvey ${ }^{2}$, J P Braybrooke ${ }^{10}$ and N A Maskell ${ }^{1,2}$

${ }^{1}$ Academic Respiratory Unit, School of Clinical Sciences, University of Bristol, Bristol BS10 5NB, UK; ${ }^{2}$ North Bristol Lung Centre, Southmead Hospital, North Bristol NHS Trust, Bristol BS10 5NB, UK; ${ }^{3}$ Applied Statistics Group, University of West of England (UWE), Bristol BS16 1OY, UK; ${ }^{4}$ Cobalt Health, Thirlestaine Road, Cheltenham GL53 7AS, UK; ${ }^{5}$ Department of Radiology, Southmead Hopsital, North Bristol NHS Trust, Bristol BS10 5NB, UK; ${ }^{6}$ Department of Radiology, Royal United Hospital Bath, Bath BA1 3NG, UK; ${ }^{7}$ Oxford Respiratory Clinical Trial Unit (Funded by the NIHR Biomedical Research Centre), Churchill Hospital, Oxford OX3 7LJ, UK; ${ }^{8}$ Department of Oncology, Royal United Hospital Bath, Bath BA1 3NG, UK; ${ }^{9}$ Department of Respiratory Medicine, Royal United Hospital Bath, Bath BA1 3NG, UK; ${ }^{10}$ Bristol Haematology and Oncology Centre, University Hospitals Bristol NHS Foundation Trust, Bristol BS2 8ED, UK; ${ }^{11}$ Department of Oncology, Cheltenham General Hospital, Cheltenham GL53 7AN, UK; ${ }^{12}$ Department of Oncology, Musgrove Park Hospital, Taunton, Severn TA1 5DA, UK and ${ }^{13}$ Department of Oncology, Great Western Hospital, Swindon SN3 6BB, UK

Background: The effect of chemotherapy on health-related quality of life (HRQoL) in malignant pleural mesothelioma (MPM) is poorly understood. Patient-individualised prognostication and prediction of treatment response from chemotherapy is useful but little evidence exists to guide practice.

Method: Consecutive patients with MPM who were fit for first-line chemotherapy with pemetrexed and cisplatin/carboplatin were recruited and followed up for a minimum of 12 months. This study focussed on the HRQoL outcomes of these patients using the EQ-5D, EORTC QLQ-C30 and LC13.

Results: Seventy-three patients were recruited of which 58 received chemotherapy and 15 opted for best supportive care (BSC). Compliance with HRQoL questionnaires was $98 \%$ at baseline. The chemotherapy group maintained HRQoL compared with the BSC group whose overall HRQoL fell $(P=0.006)$ with worsening dyspnoea and pain. The impact of chemotherapy was irrespective of histological subtype although those with non-epithelioid disease had worse HRQoL at later time points $(P=0.012)$. Additionally, those with a falling mesothelin or improvement on modified-RECIST CT at early follow-up had a better HRQoL at 16 weeks.

Conclusions: HRQoL was maintained following chemotherapy compared with a self-selected BSC group. Once chemotherapy is initiated, a falling mesothelin or improved RECIST CT findings infer a quality-of-life advantage.

Malignant pleural mesothelioma (MPM) is a heterogenous malignancy with the majority of patients following a rapidly progressive disease course. It often presents with troublesome and distressing symptoms that are difficult to control clinically and worsen with disease progression. Treatment options are limited and survival is poor, with a median life expectancy

*Correspondence: Dr D Arnold; E-mail: Arnold.dta@gmail.com

Received 17 October 2014; revised 25 January 2015; accepted 1 February 2015; published online 10 March 2015 
of 9-14 months from diagnosis (Yates et al, 1997; Chapman et al, 2008).

Randomised trial data has demonstrated survival benefits using antifolates (namely pemetrexed or raltitrexed) in combination with platinum-based cytotoxics (cisplatin or carboplatin), adding around 2 months of overall survival and reducing time to progression (Vogelzang et al, 2003). However, in the United Kingdom the proportion of patients offered chemotherapy varies widely between centres (HSCIC HaSCIC, 2014). Given the modest survival benefit, the focus of treatment remains palliative with an emphasis on preserving quality of life.

Trial data examining the impact of chemotherapy on healthrelated quality of life (HRQoL) is limited (Boons et al, 2013). Where HRQoL has been formally assessed, some studies do report an improvement in disease-specific symptoms (e.g., dyspnoea) (Bottomley et al, 2006). However, due to concurrent treatment side effects, no studies have demonstrated a benefit in overall HRQoL with chemotherapy (Muers et al, 2004). Additionally, given variable response to treatment there is an emphasis on individualised prognostic information at baseline and early disease response evaluation to inform future therapy. Currently, serial CT findings are used to assess treatment response (Byrne \& Nowak, 2004), but this approach has a no evidence base for predicting HRQoL outcomes.

The South West Area Mesothelioma and Pemetrexed (SWAMP) trial was designed to examine the relationship between baseline and serial radiological, biochemical or demographic factors and survival or chemotherapy response (Hooper et al, 2015). This substudy analyses similar factors but with a focus on HRQoL in an attempt to inform oncologists as to which patients might benefit most from chemotherapy.

\section{PATIENTS AND METHODS}

Study design. The SWAMP study is a large multi-centre prospective observational study evaluating biomarkers and radiological techniques as predictors of MPM tumour response to firstline chemotherapy (pemetrexed and cisplatin/carboplatin). The trial included a 'comparator' group of patients with similar performance status who, although deemed fit for chemotherapy, declined chemotherapeutic treatment after consultation with an oncologist. Detailed study inclusion criterion and methods can be found in the primary paper.

This sub-study focussed on the HRQoL outcomes from the SWAMP study.

HRQOL data collection. HRQoL of patients was assessed at five different time points (baseline, 6 weeks, 16 weeks, 12 months and 18 months). The patients completed the questionnaires for the first two time points (at baseline and 6 weeks) under the direct supervision of either the trial co-ordinator or the trial research nurse to ensure understanding. Subsequent questionnaires were posted to the patients to complete at home. Three different HRQoL measures were used at each time point, namely, the EQ-5D, EORTC QLQ-C30 and EORTC LC13.

The EQ-5D is a widely used preference-based generic HRQoL instrument and is the instrument favoured by NICE (National Institute of Health and Care Excellence) (Longworth \& Rowen, 2013). It measures HRQoL on 5 dimensions (mobility, self-care, usual activities, pain/discomfort and anxiety/depression) with 3 severity levels for each dimension, producing a possible 243 health states. A utility score can be generated for each health state by applying country-specific general population-elicited tariffs, which can then be used to calculate quality-adjusted life years. This data set used the UK population EQ-5D tariff (Dolan, 1997). Apart from a recently published randomised controlled trial of partial pleurectomy $v$ s talc pleurodesis, we found no other use of the EQ5D in MPM (Rintoul et al, 2014).

The EORTC QLQ-C30 (version 3) is a disease-specific questionnaire developed specifically for use in cancer. There are five functional scales (physical, role, emotional, cognitive and social), a global health item, three symptom scales (fatigue, nausea/ vomiting and pain) and single symptom items (dyspnoea, appetite loss, constipation, diarrhoea and financial difficulties). All items are converted onto a $0-100$ scale. It is important to note that higher scores for the global health and functional categories indicate higher functioning, whereas higher symptom scores denote worse symptomatology. There are a total of 30 questions in an attempt to give a broad and sensitive assessment of HRQoL in patients with cancer (Aaronson et al, 1993). It was validated for use in MPM by Nowak et al (2004) after showing predictive validity for survival and a correlation with spirometry findings.

The EORTC QLQ-LC13 was designed specifically for use in lung cancer patients undergoing chemotherapy or radiotherapy (Bergman et al, 1994). It contains 13 items that relate to lung cancer-specific symptomatology and treatment side effects, for example, cough, haemoptysis, sore mouth and peripheral neuropathy. Higher scores (from 0 to 100) denote worse symptoms. This instrument was also validated for MPM after demonstrating a strong relationship between survival and patient-reported pain (Nowak et al, 2004).

Statistical analysis. The SWAMP trial was powered for the primary end point of 'difference in time to progression on serial CT scan between patients demonstrating a metabolic response to chemotherapy on interval PET-CT vs those demonstrating no metabolic response'.

Changes across time within the various dimensions of the HRQoL instruments were assessed using Wilcoxon signed ranksum test. To minimise type I errors in the comparison of multiple factors, the level of statistical significance was set at $P=0.01$ for this analysis. Analysis of co-variance (ANCOVA) was used to assess change in HRQoL from baseline between relevant clinical subtypes. An intent-to-treat principle was used to compare the chemotherapy and comparator groups, that is, once the decision was made not to have chemotherapy (at study entry), there was no cross-over between groups at later time points. To investigate the impact of missing HRQoL data, a sensitivity analysis was performed to investigate the probability of missing values (by drop out) for significant clinical factors. Any association between clinical group and missing data was assessed using Fisher's exact test. Finally, given the previously limited use of EQ-5D in MPM, a Cox proportional hazards regression analysis was used to assess its ability to predict survival.

Trial funding and support. The trial was funded through an unrestricted educational grant from the Cobalt Appeal Fund, Cheltenham, UK.

Ethical approval and registration. Ethical and regulatory approval for the study was obtained before recruitment commenced (UK REC Reference: 08/H0102/46). The trial was registered in the national portfolio (UKCRN ID: 8450).

\section{RESULTS}

Primary study findings. The main clinical findings of the SWAMP trial have been reported in the primary paper. At baseline, a neutrophil/lymphocyte ratio of $>4$, total glycolytic volume on PET-CT of $>1800$ and non-epithelioid histology inferred a poor prognosis $(P=0.002,0.001$ and $<0.001$, respectively). Change in serial mesothelin was shown to predict disease stability on CT as well as overall survival. 
Baseline data. Between September 2008 and December 2011, 73 patients with treatment-naive pleural mesothelioma were recruited from seven different centres in the South West of England. Patients were required to be in WHO performance stage 0,1 or 2 and not have received previous chemotherapy. Of the 73 patients, 15 declined chemotherapy after discussion with their oncologist creating 'comparator' group. Table 1 summarises the baseline demographics and HRQoL of the study participants.

The comparator groups were an older cohort (median age $78 v \mathrm{~s}$ 69 years) with proportionally fewer patients with a performance status of $0(29 \%$ vs $13 \%)$. The chemotherapy and comparator groups were comparable with respect to HRQoL at baseline with no significant differences between them in any of the EQ-5D, QLQ-C30 or QLQ-LC13 dimensions. Baseline data showed that the entire cohort had significant disutility with a mean EQ-5D of 0.69 (range; -0.595 to 1 ) and QLQ-C30 global health of 65 (range, 0-100). The most marked deficiencies in the functional scales was in role function, and in the symptom scales, patients suffered from considerable fatigue, dyspnoea, insomnia and cough. Two HRQoL dimensions were shown to be baseline positive predictors of survival - a higher EQ-5D score $(P=0.026)$ and lower dyspnoea (i.e., less breathlessness) measured by the LC13 $(P=0.010)$ using Cox proportional hazards modelling.

Compliance with HRQoL questionnaires. Table 2 summarises the compliance with the HRQoL questionnaires over the course of the study.Only one questionnaire was not returned at baseline, equivalent to $98 \%$ completion but compliance fell over time. The number of forms received fell from $82 \%$ at 6 weeks to $58 \%$ at 12 months. Given natural attrition due to patient death and falling compliance, the authors decided to limit statistical analysis to visit 3 .

\section{Table 1. Baseline characteristics}

\begin{tabular}{|c|c|c|c|}
\hline & $\begin{array}{l}\text { Chemotherapy } \\
(n=58), \text { No. }(\%)\end{array}$ & $\begin{array}{c}\text { Comparator } \\
(n=15), \text { No. }(\%)\end{array}$ & $\begin{array}{c}\text { Total } \\
(n=73), \text { No. }(\%)\end{array}$ \\
\hline \multicolumn{4}{|l|}{ Age, years } \\
\hline Median & 69 & 77 & 69 \\
\hline Range & $40-83$ & $65-89$ & $40-89$ \\
\hline \multicolumn{4}{|l|}{ Sex } \\
\hline Male & $52(90)$ & $11(73)$ & 63 \\
\hline Female & $6(10)$ & $4(27)$ & 10 \\
\hline \multicolumn{4}{|l|}{ Histological subtype } \\
\hline Epithelioid & $39(67)$ & $11(73)$ & 50 \\
\hline Biphasic & $4(7)$ & $0(0)$ & 4 \\
\hline Sarcomatoid & $15(26)$ & $4(26)$ & 19 \\
\hline \multicolumn{4}{|l|}{ WHO performance status } \\
\hline 0 & 17 & 2 & 19 \\
\hline $1-2$ & 41 & 13 & 54 \\
\hline \multicolumn{4}{|l|}{ Biochemistry } \\
\hline Neutrophil/lymphocyte & 5.5 & 4.5 & 5.3 \\
\hline \multirow[t]{2}{*}{ Mesothelin } & 6.6 & 6.0 & 6.5 \\
\hline & Mean (s.d.) & Mean (s.d.) & Mean (s.d.) \\
\hline EQ-5D (range -0.595 to 1$)$ & $0.688(0.252)$ & $0.710(0.268)$ & $0.692(0.254)$ \\
\hline \multicolumn{4}{|l|}{ QLQ-C30 (range 0-100) } \\
\hline Global function & $63(21)$ & $72(17)$ & $65(20)$ \\
\hline Physical function & $74(19)$ & $73(20)$ & $73(19)$ \\
\hline Role function & $63(32)$ & $60(38)$ & $62(33)$ \\
\hline Emotional function & $77(23)$ & $87(20)$ & $79(23)$ \\
\hline Cognitive function & $82(24)$ & $82(19)$ & $82(24)$ \\
\hline Social function (range 100-0) & $71(26)$ & $73(38)$ & $71(29)$ \\
\hline Fatigue & $37(22)$ & $34(23)$ & $36(22)$ \\
\hline Pain & $26(25)$ & $18(21)$ & $24(25)$ \\
\hline $\mathrm{N}$ and $\mathrm{V}$ & $8(13)$ & $7(12)$ & $8(13)$ \\
\hline Dyspnoea & $45(29)$ & $38(21)$ & $43(28)$ \\
\hline Insomnia & $33(33)$ & $19(28)$ & $30(33)$ \\
\hline Appetite loss & $23(29)$ & $36(39)$ & $25(32)$ \\
\hline Constipation & $18(27)$ & $27(34)$ & $20(29)$ \\
\hline Diarrhoea & $6(16)$ & $4(11)$ & $6(15)$ \\
\hline Financial problems & $16(29)$ & $7(19)$ & $14(28)$ \\
\hline \multicolumn{4}{|l|}{ QLQ-LC13 (range 100-0) } \\
\hline Dyspnoea & $33(23)$ & $36(18)$ & $34(22)$ \\
\hline Coughing & $31(22)$ & $27(26)$ & $30(23)$ \\
\hline Haemoptysis & $2(10)$ & $0(0)$ & $1(9)$ \\
\hline Sore mouth & $4(13)$ & $0(0)$ & $3(11)$ \\
\hline Dysphagia & $7(16)$ & $4(12)$ & $6(16)$ \\
\hline Peripheral neuropathy & $6(16)$ & $7(19)$ & $6(16)$ \\
\hline Alopecia & $2(10)$ & $2(9)$ & $2(10)$ \\
\hline Pain in chest & $22(26)$ & $24(32)$ & $23(27)$ \\
\hline Pain in arm & $18(25)$ & $13(17)$ & $17(24)$ \\
\hline Pain (other) & $18(26)$ & $10(16)$ & $16(25)$ \\
\hline
\end{tabular}


Table 2. Compliance with HRQoL questionnaires

\begin{tabular}{|c|c|c|c|}
\hline & $\begin{array}{l}\text { Chemotherapy } \\
(n=58), \text { No. }(\%)\end{array}$ & $\begin{array}{c}\text { Comparator } \\
(n=15), \text { No. }(\%)\end{array}$ & $\begin{array}{c}\text { Total } \\
(n=73), \text { No. }(\%)\end{array}$ \\
\hline \multicolumn{4}{|l|}{ Visit 1 - baseline } \\
\hline $\begin{array}{l}\text { Expected no. of forms } \\
\text { Received no. of forms }\end{array}$ & $\begin{array}{c}58 \\
57(98.3)\end{array}$ & $\begin{array}{c}15 \\
15(100)\end{array}$ & $\begin{array}{c}73 \\
72(98.6)\end{array}$ \\
\hline \multicolumn{4}{|l|}{ Visit 2-6 weeks } \\
\hline $\begin{array}{l}\text { Expected no. of forms } \\
\text { Received no. of forms }\end{array}$ & $\begin{array}{c}58 \\
45(77.6)\end{array}$ & $\begin{array}{c}15 \\
15(100)\end{array}$ & $\begin{array}{c}73 \\
60(82.2)\end{array}$ \\
\hline \multicolumn{4}{|l|}{ Visit 3-16 weeks } \\
\hline $\begin{array}{l}\text { Expected no. of forms } \\
\text { Received no. of forms }\end{array}$ & $\begin{array}{c}52 \\
42(80.8)\end{array}$ & $\begin{array}{c}15 \\
12(80.0)\end{array}$ & $\begin{array}{c}67 \\
54(80.6)\end{array}$ \\
\hline \multicolumn{4}{|l|}{ Visit 4-12 months } \\
\hline $\begin{array}{l}\text { Expected no. of forms } \\
\text { Received no. of forms }\end{array}$ & $\begin{array}{c}45 \\
26(57.8)\end{array}$ & $\begin{array}{c}12 \\
7(58.3)\end{array}$ & $\begin{array}{c}57 \\
33(57.9)\end{array}$ \\
\hline \multicolumn{4}{|l|}{ Visit 5-18 months } \\
\hline $\begin{array}{l}\text { Expected no. of forms } \\
\text { Received no. of forms }\end{array}$ & $\begin{array}{c}31 \\
24(77.4)\end{array}$ & $\begin{array}{c}10 \\
5(50.0)\end{array}$ & $\begin{array}{c}41 \\
29(70.7)\end{array}$ \\
\hline
\end{tabular}

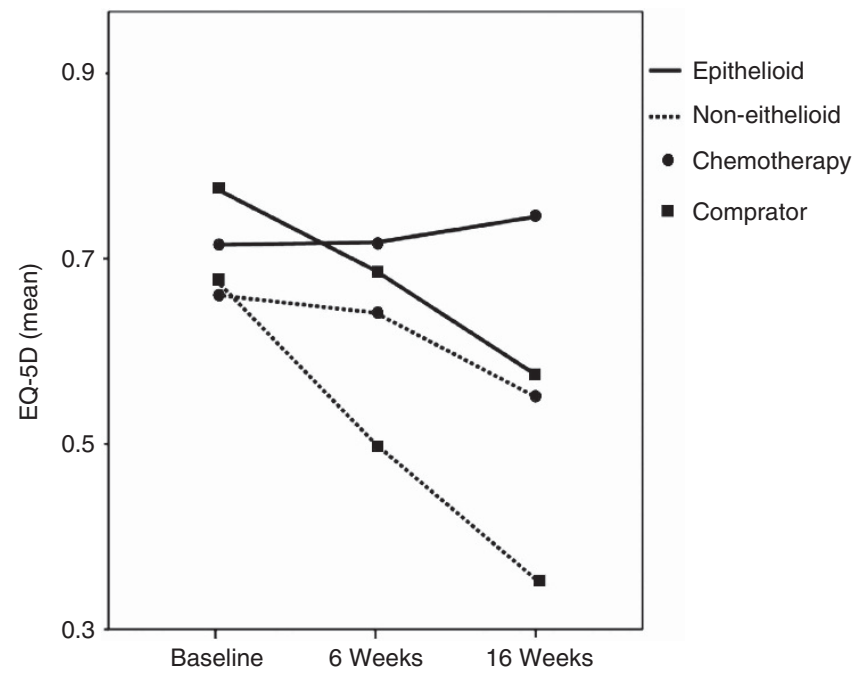

Figure 1. Graph showing the change in mean EQ-5D between the chemotherapy and comparator groups divided by histological subtype.

Chemotherapy vs comparator group. There were significant differences between the chemotherapy and comparator groups over time, despite comparable HRQoL at baseline. Most notably in overall health measured by the EQ-5D where the chemotherapy group maintained overall health throughout whereas the comparator group worsened considerably, so that by time point 3 the difference between them using the ANCOVA approach was significant $(P=0.006)$. Figure 1 demonstrates the change in mean EQ-5D from baseline to 16 weeks between the chemotherapy and comparator arms divided by histological subtype. For the EORTC instruments, the Wilcoxon signed ranked test was used to investigate change in functional and symptom scales from baseline. Any dimensions that changed significantly $(P<0.01)$ are represented graphically in Figure 2. General worsening of global health, physical function and fatigue were seen across the cohort. At visit 2 , the chemotherapy group experienced worsening social function as well as worsening symptoms of nausea and vomiting and sore mouth. At visit 3, these functional and symptoms scores had resolved but alopecia had worsened significantly. The comparator group did not suffer from the same gastrointestinal symptoms or alopecia but, at visit 3 , had significantly worse dyspnoea and arm pain compared with baseline.

Chemotherapy group. The following analyses use only the chemotherapy arm $(n=58)$. Table 3 shows the effect of demographic, tumour and biochemical factors on HRQoL. This analysis focussed on results from the EQ-5D. An independent samples $T$-test showed no significant differences between the groups at baseline. An ANCOVA method was used to assess the change in HRQoL from baseline to 16 weeks. Those with nonepithelioid histology had a decline in HRQoL and the variance from baseline between the groups was significant, although the same pattern was seen in the comparator group (see Figure 1). Serum mesothelin was measured at baseline and visit 2, a rising or falling mesothelin was not subject to any thresholds. Patients with a falling mesothelin at visit 2 compared with baseline had an improvement in HRQoL compared with those with a rising mesothelin (see Figure 3). Disease response on serial CT imaging (using RECIST criteria) at 8 weeks was also positively correlated with HRQoL (see Figure 4).

Sensitivity analysis. The sensitivity analysis showed no differences in the proportion of missing results between any of the clinical groups tested, supporting our main findings. Clinical groups examined included chemotherapy vs comparator $(P=0.75)$, age $>70$ vs age $<70(P=0.09)$, histology $(P=0.22)$, baseline EQ-5D value $\geqslant 0.7$ vs $<0.7(P=1.00)$ and serum mesothelin falling $v s$ rising at visit $2(P=1.00)$.

\section{DISCUSSION}

The aim of this study was to investigate the effect of chemotherapy on HRQoL in MPM, where scanty literature exists to inform clinicians. HRQoL was formally assessed using a variety of diseasespecific and generic instruments. The EQ-5D is a generic HRQoL instrument recommended by NICE for use in clinical trials but has no published use in chemotherapy for MPM. The EQ-5D was able to select out clinically distinct sub-groups and was associated with overall survival and as such forms the basis of our more detailed analysis.

Despite being advocated by NICE (NICE, 2008), the use of chemotherapy for MPM is variable between centres across the UK (HSCIC HaSCIC, 2014), due mainly to uncertainty about its overall benefit. Pemetrexed and cisplatin is the only approved 
A

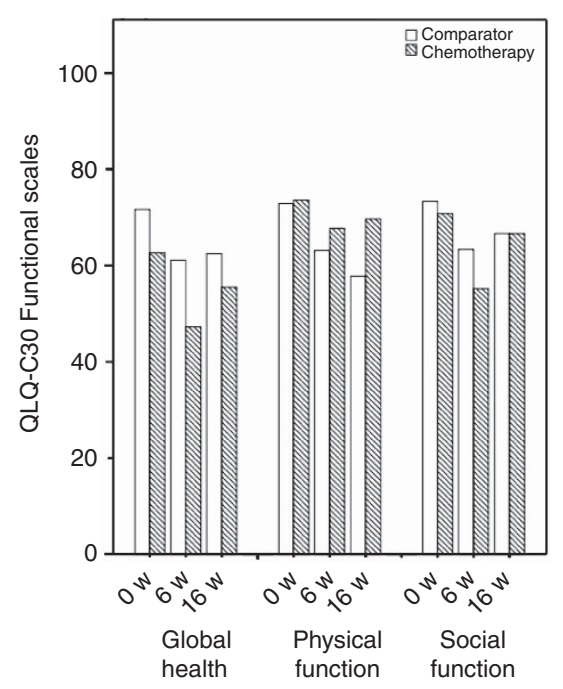

B

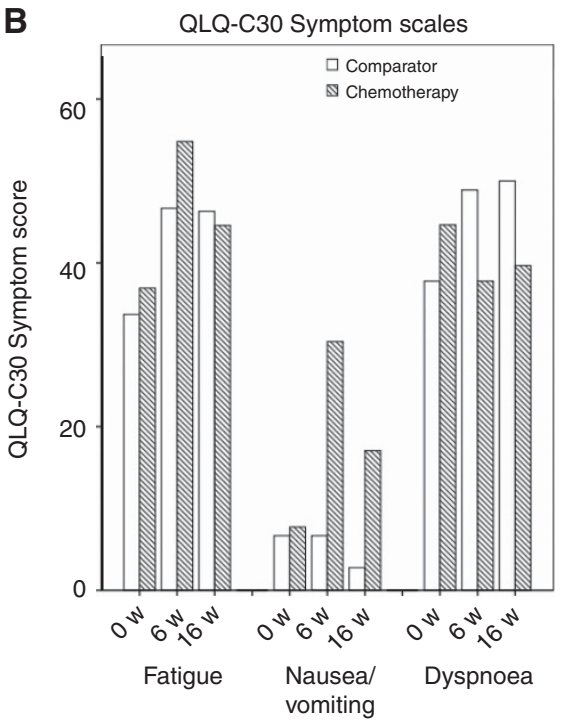

C

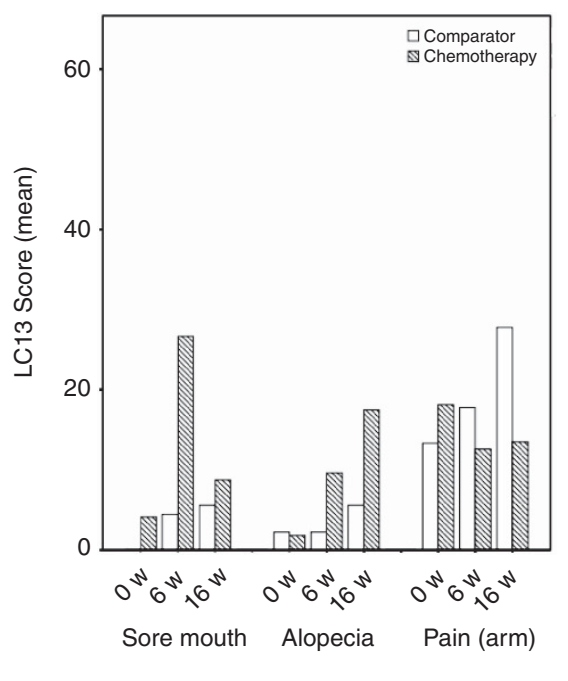

Figure 2. HRQoL dimensions with significant $(P<0.01)$ changes from baseline between the chemotherapy and comparator groups. $(A)$ QLO-C30 Functional Scales. (B) QLQ-C30 Symptom Scales. (C) LC13 Symptom Scales.

\begin{tabular}{|c|c|c|c|}
\hline \multirow{2}{*}{$\begin{array}{l}\text { Factor } \\
\text { Age }>70 \text { years }(n=20) \\
\text { vs } \\
\text { Age } \leqslant 70 \text { years }(n=22)\end{array}$} & \multirow{2}{*}{$\begin{array}{c}\text { Mean EQ-5D } \\
\text { at baseline }\end{array}$} & \multicolumn{2}{|c|}{ Change in EQ-5D from baseline to 16 weeks (ANCOVA) } \\
\hline & & $\begin{array}{l}+0.036 \\
-0.047\end{array}$ & $(P=0.234)$ \\
\hline $\begin{array}{l}\text { Epithelioid histology }(n=31) \\
\text { vs } \\
\text { Nonepithelioid histology }(n=11)\end{array}$ & $\begin{array}{l}0.715 \\
0.661\end{array}$ & $\begin{array}{r}+0.032 \\
-0.110\end{array}$ & $(P=0.012)$ \\
\hline $\begin{array}{l}\text { Falling mesothelin at } 6 \text { weeks }(n=18) \\
\text { vs } \\
\text { Rising mesothelin at } 6 \text { weeks }(n=22)\end{array}$ & $\begin{array}{l}0.662 \\
0.736\end{array}$ & $\begin{array}{l}+0.085 \\
-0.086\end{array}$ & $(P=0.049)$ \\
\hline $\begin{array}{l}\text { Neutrophi//leucocyte ratio }<4(n=22) \\
\text { vs } \\
\text { Neutrophil/leucocyte ratio }>4(n=18)\end{array}$ & $\begin{array}{l}0.719 \\
0.672\end{array}$ & $\begin{array}{r}-0.053 \\
+0.045\end{array}$ & $(P=0.291)$ \\
\hline $\begin{array}{l}\text { Disease improvement on CT at } 8 \text { weeks }(n=6) \\
\text { vs } \\
\text { Disease stable/progression on CT at } 8 \text { weeks }(n=35)\end{array}$ & $\begin{array}{l}0.745 \\
0.685\end{array}$ & $\begin{array}{l}+0.119 \\
-0.027\end{array}$ & $(P=0.045)$ \\
\hline
\end{tabular}

therapy following the results of a phase III clinical trial by Vogelzang et al (2003) showing a survival benefit for combination chemotherapy vs cisplatin alone (12.1 vs 9.3 months). Given that the improvement in survival is small and the symptomatology from MPM is severe, the focus of treatment remains palliative.

The majority of previous studies formally assessing HRQoL in MPM have used the EORTC QLQ-C30 and/or LC13. Baseline data from all studies demonstrates that patients are often in poor health at study entry. Bottomley et al (2006) used these instruments in 250 patients receiving either raltitrexed and cisplatin or cisplatin alone. Mean global health at baseline was around 55 (range 0-100) and patients were impaired in all functional dimensions, particularly role function, a characteristic of our cohort's baseline. The Bottomley and SWAMP cohorts were also comparable with respect to symptom scales at baseline with significant fatigue, pain, dyspnoea, insomnia and appetite loss. An RCT of video-assisted thorascopic partial pleurectomy vs pleurodesis (MesoVATS trial) is the only other published use of the EQ-5D in mesothelioma. Interestingly, despite also using the EORTC QLQ-C30 and LC13, the significant HRQoL findings were only apparent when analysing the EQ-5D results (Rintoul et al, 2014).
As our study is not a randomised controlled trial, any conclusions made comparing the differences between a chemotherapy and self-selected comparator arm must be interpreted with caution. The potential confounders of self-selection are numerous. It is conceivable that the non-chemotherapy group were not suffering from such distressing symptoms at baseline and felt that the potential chemotherapy side effects outweighed the small survival benefit. Such a confounder would significantly effect HRQoL measures. Despite this, at baseline the groups were similar with respect to overall quality of life and symptomatology. The chemotherapy group reported predictable chemotherapy side effects of nausea and vomiting, sore mouth and alopecia, all of which have been reported in similar analyses (Bottomley et al, 2006). It is worth noting that the majority of performance and symptom scales had improved by 16 weeks when treatment had finished. Symptoms that persisted at 16 weeks have all been previously associated with cisplatin, namely fatigue and alopecia (Flechtner \& Bottomley, 2003). The chemotherapy group reported improvement in the symptoms of dyspnoea and arm pain, which worsened significantly in the comparator group. Most notably, the overall HRQoL assessed using the EQ-5D remained stable in the 


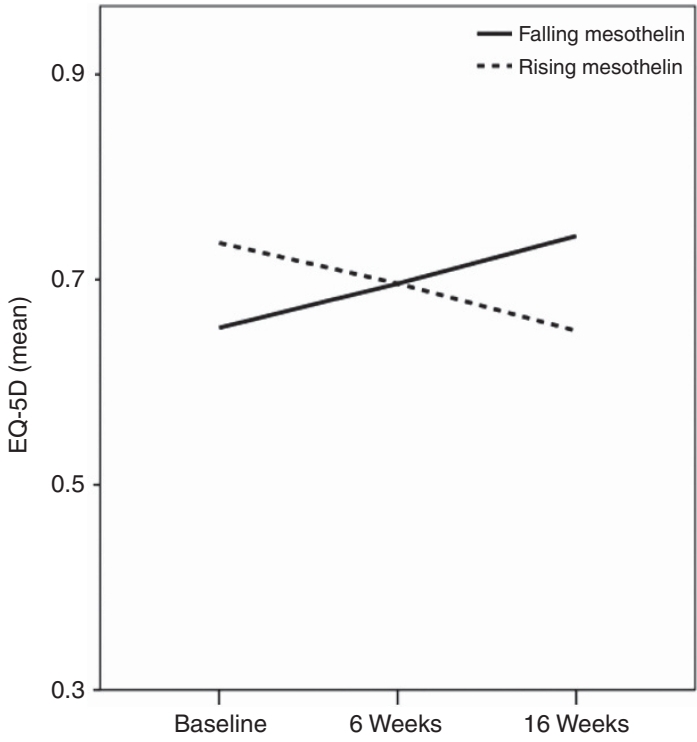

Figure 3. Change in mean EQ-5D values in patients receiving chemotherapy with a falling vs rising mesothelin at 6 weeks.

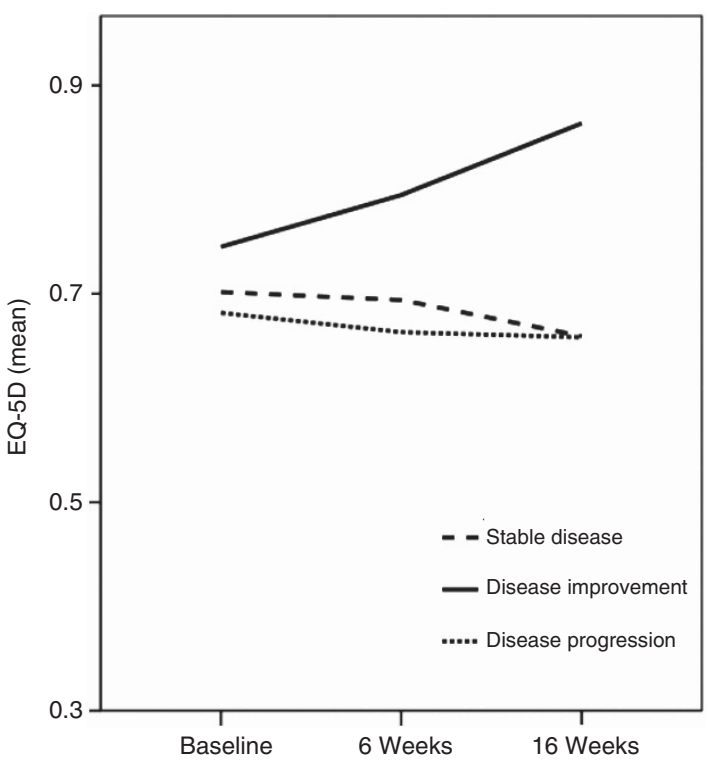

Figure 4. Change in mean EQ-5D values in patients receiving chemotherapy divided by CT finding at 8 weeks.

chemotherapy group but declined significantly in the comparator arm. At 16 weeks, the difference between the two was 0.20 , greater than the minimally important difference for lung cancer published in the literature (Pickard et al, 2007). No EQ-5D literature exists for comparison and few studies formally assessing HRQoL have a comparator arm where no chemotherapy was used. Muers et al (2004) randomised 409 patients into three separate groups, including one group where no chemotherapy was given and two others given mitomycin, vinblastine and cisplatin \pm vinorelbine. Patients in this trial completed the QLQ-C30 and LC13 every 3 weeks up to 21 weeks. The investigators concluded that there was no significant difference in quality of life between the groups. Bottomley et al (2006) also found no difference between a raltitrexed and cisplatin $v s$ cisplatin alone regimen with respect to overall HRQoL but did note that dyspnoea was improved in the dual treatment arm.
In previous studies, patients with non-epithelioid disease had poorer response to chemotherapy and survival (Neumann et al, 2004; Musk et al, 2011), but no study has linked histology to HRQoL outcomes. We saw a significant decline in overall HRQoL measured with the EQ-5D in patients with non-epithelioid histology compared with epithelioid disease, from comparable baselines. Despite the concurrent use of two disease-specific instruments alongside the EQ-5D, it is not clear what symptomatology contributes to this effect. Although the non-epithelioid patients suffer from worse fatigue, cough and chest pain at 16 weeks, none of these differences are statistically significant. The effect on overall HRQoL is likely to be a culmination of factors that, due to smaller numbers of non-epithelioid disease and complex symptomatology, this analysis cannot detect. Additionally, this analysis found no evidence that chemotherapy was less effective in non-epithelioid MPM.

Once chemotherapy is initiated, early markers of prognostication and future HRQoL are useful for oncologists given variable treatment response and side effect profile between patients. Serial CT scanning using the modified RECIST criteria has been a predictor of survival in previous studies and is often used to inform future therapy but has never been correlated with later HRQoL. We found that patients who had responded to chemotherapy at an 8 -week CT scan had better HRQoL at later time points. However, this only applied to a small proportion of the patients $(17 \%, n=6$ out of 35) Additionally, these time-consuming investigations require expert interpretation and can be made more difficult to interpret if previous surgery or pleurodesis has been performed (Kwek et al, 2004). Several serum blood tests to augment radiological data have been trialled, with variable success. Serum mesothelin has been shown to be a useful biomarker of treatment response. Mesothelin is a cell adhesion glycoprotein present on the surface of mesothelial cells and is overexpressed in several malignancies, including MPM (Creaney \& Robinson, 2005). An early (2 months) falling mesothelin has been correlated with survival and radiological treatment response (primary SWAMP study) (Grigoriu \& Scherpereel, 2008; Creaney et al, 2011), but has never previously been linked to HRQoL. Kao et al (2013) found that baseline VEGF, NLR and CRP all correlated with dimensions in the Lung Cancer Symptom Scale. Unfortunately, due to limited longitudinal data they could not make any conclusions about changes in inflammatory markers and response to treatment. Mesothelin is a simple serum blood test which, if tested at an early clinic appointment, could be used as a proxy for both better survival and maintenance of quality of life.

This study had several potential limitations that might weaken our conclusions. As previously stated, the comparator group is comprised of a small group of patients who although deemed fit for chemotherapy declined it after an informed discussion with a consultant oncologist. As they are self-selected, they cannot be classed as a control group. They had similar performance and HRQoL status at baseline but are older (although increasing age was not related to poor HRQoL in this cohort) with a higher proportion of females. Our finding that HRQoL is maintained in the chemotherapy group compared with the comparator group should be cautiously interpreted. Second, when analysing several HRQoL instruments with numerous dimensions there is an increased risk of type I errors. We therefore set a $P$-value of $<0.01$ when assessing the QLQ-C30 and LC13 instruments, which reduces the sensitivity of the analysis but strengthens the conclusions. Finally, although a cohort of 73 patients suffering from a rare disease is a good sample size, there was significant attrition in questionnaire completion due to patient death and falling compliance. To avoid survival bias and the bias introduced by only including patients well enough to complete questionnaires, we limited our analysis to time point 3 (16 weeks). At this point, an assessment can be made of postchemotherapy functional status with predicted resolution of chemotherapy-associated side effects. 
In summary, this study robustly assessed HRQoL in patients receiving chemotherapy for MPM. Three validated questionnaires were used with good compliance rates at baseline, during and after chemotherapy, making this one of the most comprehensive assessments of HRQoL in MPM literature. Based on this analysis, patients who receive chemotherapy have better HRQoL outcomes at 16 weeks compared with a self-selected comparator group, with better dyspnoea and pain scores. In patients undergoing chemotherapy, those with non-epithelioid histology have worse overall HRQoL at later time points but this is likely due to more aggressive disease. A falling mesothelin after two cycles of chemotherapy predicts improved quality of life and was seen in $44 \%$ of patients. Disease response on CT also predicts improved quality of life but was observed more infrequently in this series. Clinicians can be confident in the HRQoL benefits of continued chemotherapy given either of these favourable markers at an early stage of treatment.

\section{CONFLICT OF INTEREST}

The authors declare no conflict of interest.

\section{REFERENCES}

Aaronson NK, Ahmedzai S, Bergman B, Bullinger M, Cull A, Duez NJ, Filiberti A, Flechtner H, Fleishman SB, de Haes JC, Kaasa S, Klee M, Osoba D, Razavi D, Rofe PB, Schraub S, Sneeuw K, Sullivan M, Takeda F (1993) The European Organization for Research and Treatment of Cancer QLQ-C30: a quality-of-life instrument for use in international clinical trials in oncology. J Natl Cancer Inst 85(5): 365-376.

Bergman B, Aaronson NK, Ahmedzai S, Kaasa S, Sullivan M (1994) The EORTC QLQ-LC13: a modular supplement to the EORTC Core Quality of Life Questionnaire (QLQ-C30) for use in lung cancer clinical trials. EORTC Study Group on Quality of Life. Eur J Cancer 30A(5): 635-642.

Boons CC, VANT MW, Burgers JA, Beckeringh JJ, Wagner C, Hugtenburg JG (2013) The value of pemetrexed for the treatment of malignant pleural mesothelioma: a comprehensive review. Anticancer Res 33(9): 3553-3561.

Bottomley A, Gaafar R, Manegold C, Burgers S, Coens C, Legrand C, Vincent M, Giaccone G, Van Meerbeeck J. Group EL-C, National Cancer Institute C (2006) Short-term treatment-related symptoms and quality of life: results from an international randomized phase III study of cisplatin with or without raltitrexed in patients with malignant pleural mesothelioma: an EORTC Lung-Cancer Group and National Cancer Institute, Canada, Intergroup Study. J Clin Oncol 24(9): 1435-1442.

Byrne MJ, Nowak AK (2004) Modified RECIST criteria for assessment of response in malignant pleural mesothelioma. Ann Oncol 15(2): 257-260.

Chapman A, Mulrennan S, Ladd B, Muers MF (2008) Population based epidemiology and prognosis of mesothelioma in Leeds, UK. Thorax 63(5): 435-439.

Creaney J, Francis RJ, Dick IM, Musk AW, Robinson BW, Byrne MJ, Nowak AK (2011) Serum soluble mesothelin concentrations in malignant pleural mesothelioma: relationship to tumor volume, clinical stage and changes in tumor burden. Clin Cancer Res 17(5): 1181-1189.

Creaney J, Robinson BW (2005) Detection of malignant mesothelioma in asbestos-exposed individuals: the potential role of soluble mesothelinrelated protein. Hematol Oncol Clin North Am 19(6): 1025-1040v.

Dolan P (1997) Modeling valuations for EuroQol health states. Med Care 35(11): 1095-1108.
Flechtner H, Bottomley A (2003) Fatigue and quality of life: lessons from the real world. Oncologist 8(Suppl 1): 5-9.

Grigoriu BD, Scherpereel A (2008) Diagnostic value of soluble mesothelin in malignant mesothelioma. Thorax 63(1): 87-88author reply 87.

Hooper CE, Lyburn I, Searle J, Darby M, Hall T, Hall D, Morley A, White P, Rahman N, De Winton E, Clive A, Masani V, Arnold D, Dangoor A, Guglani S, Jankowska P, Lowndes S, Harvey JE, Braybrooke J, Maskell NA (2015) The South West Area Mesothelioma and Pemetrexed (SWAMP) trial - A multi-centre prospective observational study evaluating novel markers of chemotherapy response and prognostication. Br J Cancer.

HSCIC HaSCIC (2014) National Lung Cancer Audit Report 2014 (Mesothelioma).

Kao SC, Vardy J, Harvie R, Chatfield M, van Zandwijk N, Clarke S, Pavlakis N (2013) Health-related quality of life and inflammatory markers in malignant pleural mesothelioma. Support Care Cancer 21(3): 697-705.

Kwek BH, Aquino SL, Fischman AJ (2004) Fluorodeoxyglucose positron emission tomography and CT after talc pleurodesis. Chest 125(6): 2356-2360.

Longworth L, Rowen D (2013) Mapping to obtain EQ-5D utility values for use in NICE health technology assessments. Value Health 16(1): 202-210.

Muers MF, Rudd RM, O’Brien ME, Qian W, Hodson A, Parmar MK, Girling DJ. British Thoracic Society Mesothelioma G (2004) BTS randomised feasibility study of active symptom control with or without chemotherapy in malignant pleural mesothelioma: ISRCTN 54469112. Thorax 59(2): 144-148.

Musk AW, Olsen N, Alfonso H, Reid A, Mina R, Franklin P, Sleith J, Hammond N, Threlfall T, Shilkin KB, de Klerk NH (2011) Predicting survival in malignant mesothelioma. Eur Respir J 38(6): 1420-1424.

Neumann V, Rutten A, Scharmach M, Muller KM, Fischer M (2004) Factors influencing long-term survival in mesothelioma patients-results of the German mesothelioma register. Int Arch Occup Environ Health 77(3): 191-199.

NICE (2008) Pemetrexed for the treatment of malignant pleural mesothelioma. NICE Technology Appraisal Guidance 135.

Nowak AK, Stockler MR, Byrne MJ (2004) Assessing quality of life during chemotherapy for pleural mesothelioma: feasibility, validity, and results of using the European Organization for Research and Treatment of Cancer Core Quality of Life Questionnaire and Lung Cancer Module. J Clin Oncol 22(15): 3172-3180.

Pickard AS, Neary MP, Cella D (2007) Estimation of minimally important differences in EQ-5D utility and VAS scores in cancer. Health Qual Life Outcomes 5: 70.

Rintoul RC, Ritchie AJ, Edwards JG, Waller DA, Coonar AS, Bennett M, Lovato E, Hughes V, Fox-Rushby JA, Sharples LD. MesoVatsCollaborators (2014) Efficacy and cost of video-assisted thoracoscopic partial pleurectomy versus talc pleurodesis in patients with malignant pleural mesothelioma (MesoVATS): an open-label, randomised, controlled trial. Lancet 384(9948): 1118-1127.

Vogelzang NJ, Rusthoven JJ, Symanowski J, Denham C, Kaukel E, Ruffie P, Gatzemeier U, Boyer M, Emri S, Manegold C, Niyikiza C, Paoletti P (2003) Phase III study of pemetrexed in combination with cisplatin versus cisplatin alone in patients with malignant pleural mesothelioma. J Clin Oncol 21(14): 2636-2644.

Yates DH, Corrin B, Stidolph PN, Browne K (1997) Malignant mesothelioma in south east England: clinicopathological experience of 272 cases. Thorax 52(6): 507-512.

This work is licensed under the Creative Commons national License. To view a copy of this license, visit http://
nat creativecommons.org/licenses/by-nc-sa/4.0/ 